
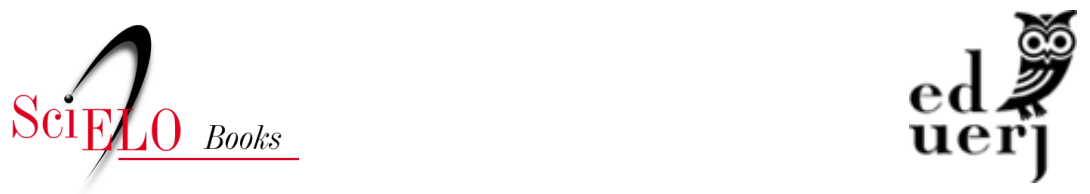

\title{
Capitulo 5 - Atenção Básica: a linha que costura o cuidado ao sujeito com obesidade
}

\author{
Erika Cardoso dos Reis \\ Luciana da Silva Rodriguez \\ Phillipe Augusto Ferreira Rodrigues
}

\section{SciELO Books / SciELO Livros / SciELO Libros}

REIS, E. C., RODRIGUEZ, L. S., and RODRIGUES, P. A. F. Atenção Básica: a linha que costura o cuidado ao sujeito com obesidade. In.: SILVA, A. C. F., MOTTA, A. L. B., and CASEMIRO, J. P., eds. Alimentação e nutrição na atenção básica: reflexões cotidianas e contribuições para prática do cuidado [onine]. Rio de Janeiro: EDUERJ, 2021, pp 99-123. ISBN: 978-65-87949-11-6. https://doi.org/10.7476/9786587949116.0006.

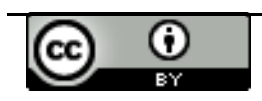

All the contents of this work, except where otherwise noted, is licensed under a Creative Commons Attribution 4.0 International license.

Todo o conteúdo deste trabalho, exceto quando houver ressalva, é publicado sob a licença Creative Commons Atribição 4.0.

Todo el contenido de esta obra, excepto donde se indique lo contrario, está bajo licencia de la licencia Creative Commons Reconocimento 4.0. 


\title{
Capitulo 5 \\ Atenção Básica: a linha que costura o cuidado ao sujeito com obesidade
}

\author{
Erika Cardoso dos Reis \\ Luciana da Silva Rodriguez \\ Phillipe Augusto Ferreira Rodrigues
}

Ao longo dos últimos anos, observa-se o crescimento acentuado e progressivo da obesidade na população brasileira, demandando açóes de cuidado em saúde prioritárias no âmbito da Atenção Básica (AB). Segundo dados da Organização Mundial de Saúde (2000; 2012), a obesidade tem tomado proporçốes epidêmicas. A prevalência mundial duplicou no intervalo de 1980 e 2008, passando a acometer $10 \%$ da população masculina e $15 \%$ da feminina, afetando quase 500 milhôes de adultos maiores de 20 anos (WHO, 2012).

Ao comparar os dados sobre estado nutricional da populaçáo brasileira estimados em cinco inquéritos com abrangência nacional: Estudo Nacional da Despesa Familiar (ENDEF), 1974-75; Pesquisa Nacional sobre Saúde e Nutrição (PNSN), 1989; Pesquisa de Orçamentos Familiares (POF), 2002-03; POF, 2008-09; e Pesquisa Nacional sobre Saúde (PNS), 2013, a prevalência do déficit de peso em adultos declinou de modo contínuo, do primeiro estudo ao mais recente, e houve um aumento vertiginoso do excesso de peso e obesidade. Em homens, o déficit de peso variou de 8,0\%, em 1974-75, para 4,4\%, em 1989, deste para 3,1\%, em 2002-03, 1,8\%, em 2008-09, e 1,9\%, em 2013. Em mulheres, de 11,8\%, em 1974-75, para 6,4\%, em 1989, deste para 5,6\%, em 2002-03, 3,6\%, em 2008-09, e 2,5\%, em 2013. 
Os dados mais recentes no Brasil sobre a obesidade são do Ministério da Saúde (MS) a partir da Vigilância de Fatores de Risco e Proteção para Doenças Crônicas por Inquérito Telefônico (VIGITEL), que vem sendo realizado anualmente desde 2006. No ano de 2018, a frequência de excesso de peso foi de $57,8 \%$ entre os homens e 53,9\% entre as mulheres, e a frequência de obesidade, 18,7\% entre os homens e $20,7 \%$ entre as mulheres, além disso, a prevalência duplica na população acima de 25 anos. $\mathrm{Na}$ análise do período de 2006 a 2018, a proporção de pessoas com obesidade (IMC $\geq 30 \mathrm{~kg} /$ $\mathrm{m}^{2}$ ) cresceu mais de 70\%, passando de $11,4 \%$, em 2006, para 19,8\%, em 2016 (Brasil, 2019). Esses números são alarmantes tendo em vista que a obesidade tem sido reconhecida como um dos fatores de maior risco para o adoecimento de adultos (Brasil, 2014a).

Considerando este cenário e o desafio do cuidado das pessoas com condiçôes crônicas e multimorbidades, a proposta deste capítulo é discutir, dentro da organização da Linha de Cuidado (LC) da obesidade, os desafios e as possibilidades das ações da Atenção Básica (AB).

\section{Juntando os fios e as agulhas: costurando conceitos}

Segundo a Organização Mundial de Saúde (OMS), a obesidade é definida como um agravo de caráter multifatorial decorrente de balanço energético positivo que favorece o acúmulo de gordura, associado a riscos para a saúde, devido à sua relação com complicaçóes metabólicas, como o aumento da pressão arterial, dos níveis de colesterol e triglicerídeos sanguíneos e resistência à insulina. Entre suas causas, estão relacionados fatores biológicos, históricos, ecológicos, econômicos, sociais, culturais e políticos (WHO, 2000). Ela também está incluída no grupo das Doenças Crônicas Não Transmissíveis (DCNT) e é um fator de risco para outras morbidades, como hipertensão arterial sistêmica, diabetes mellitus tipo II, dislipidemia, hipotireoidismo e alguns tipos de câncer. Toda essa multiplicidade sobrecarrega o sistema de saúde 
com uma demanda crescente de doenças correlacionadas, o que traz repercussões importantes nos custos das ações de média e alta complexidade no tratamento não apenas da obesidade, mas também das doenças a ela associadas (Bahia e Araújo, 2014; Oliveira, 2013).

Atualmente, o critério mais utilizado para avaliação da obesidade na prática clínica e em nível populacional é o Índice de Massa Corporal (IMC), que apresenta boa correlação com desenvolvimento de doenças crônicas e mortalidade (Calle et al., 1999; Brasil, 2014a). Esse critério é muito utilizado devido à simplicidade dos recursos utilizados: balança e estadiômetro, presentes em quaisquer unidades básicas de saúde. Outros métodos, apesar de mais precisos, incluiriam custo elevado e dificuldades técnicas para seu treinamento e sua execução. A classificação com base no IMC e seus respectivos valores estão apresentados na tabela 1. Sua elaboração segue a OMS (1995), acrescida da designação superobesidade para IMC maior que $50 \mathrm{~kg} / \mathrm{m}^{2}$, definida pela American Society for Bariatric Surgery (1997).

Tabela 1. Classificação do estado nutricional de acordo com o IMC

\begin{tabular}{lc}
\hline Classificaçáo & Índice de Massa Corporal $\left(\mathbf{k g} / \mathbf{m}^{2}\right)$ \\
\hline Abaixo do peso & $<18,5$ \\
\hline Peso normal/adequado & $18,5-24,9$ \\
\hline Sobrepeso & $25-29,9$ \\
\hline Obesidade grau I & $30-34,9$ \\
\hline Obesidade grau II & $35-39,9$ \\
\hline Obesidade grau III & $\geq 40$ \\
\hline Superobesidade & $>50$ \\
\hline
\end{tabular}

Fonte: WHO,1995; American Society for Bariatric Surgery, 1997.

É importante ressaltar que a etiologia da obesidade é complexa e multifatorial, resultado da interação de questôes biológicas, 
ambientais, econômicas, sociais, culturais e políticas (Brasil, 2014a; WHO, 2000). Esse conjunto de fatores confere à obesidade o status de grande problema de saúde pública, o que tem preocupado autoridades mundiais e as mobilizado para a construção de LC para essa condição. Isso significa instituir diretrizes que definam fluxos assistenciais para a atenção à saúde, promoção, prevenção, assistência e reabilitação. Para isso, é necessária uma rede que acione diversos setores e atores de forma a impactar positivamente no processo saúde-doença e, nesse cenário a $\mathrm{AB}$ tem papel de protagonista.

Barbara Starfield (2002) destaca que países com os sistemas de saúde orientados pela Atenção Primária à Saúde ${ }^{1}$ apresentam maiores chances de alcançar melhores níveis de saúde com custos mais baixos. "É a atenção que organiza e racionaliza o uso de todas as tecnologias e recursos, tanto básicos como especializados, direcionados para a promoção, manutenção e melhora da saúde" (Starfield, 2002, p. 28).

Merhy e Feuerwerker (2009) discutem a construção das "caixas de ferramentas tecnológicas" utilizadas pelos profissionais e mostram como a combinação dessas tecnologias compóem as diferentes ações de cuidado em saúde nos diferentes pontos de atenção. Os autores distinguem três tipos: as duras, as leve-duras e as leves. Cada uma delas tem uma densidade e custos diferentes, o que confere características a cada nível de atenção. As primeiras, tecnologias duras, correspondem aos equipamentos, exames, medicamentos que compóem os procedimentos nas intervençóes terapêuticas. As leve-duras englobam os saberes envolvidos na formação do profissional, que lhe fornece um determinado ponto de vista sobre a avaliação de cada caso. Está relacionada a um aspecto endurecido, mas que é flexibilizado pela

1 Destaca-se que, neste capítulo, os autores seguem a Política Nacional de Atenção Básica que considera os termos "Atenção Básica" e "Atenção Primária à Saúde" como equivalentes, conforme pode ser verificado em: BRASIL. Ministério da Saúde. Politica Nacional de Atenção Básica. Ministério da Saúde, 2012; e na atual revisão formalizada pela Portaria n. ${ }^{2}$.436, de 21 de setembro de 2017. 
interação com o usuário. E a última tecnologia, a chamada de leve, trata dos construtos produzidos nas relações envolvidas no encontro trabalhador-usuário, como a escuta, o interesse, a construção de vínculos e o estabelecimento de confiança.

A definição de Starfield (2002) revela que a caixa de ferramentas utilizadas na $\mathrm{AB}$ confere um maior peso às tecnologias leves. Cabe à $\mathrm{AB}$ estar atenta às especificidades locais, aos determinantes sociais e às singularidades dos casos que enriquecem e ampliam o raciocínio clínico do profissional, que deve ter como pressuposto básico de sua atuação o cuidado e respeito com os sujeitos. A autora também destaca que um sistema tem maior impacto na saúde se utiliza bem não só os recursos de tecnologia dura, mas principalmente se consegue usar de forma ampla as tecnologias leves.

No Brasil, o MS apresenta formalmente, a partir de 1994, a Estratégia de Saúde da Família (ESF) como uma das estratégias prioritárias no país para reorganização dos serviços de saúde, a partir da transição de um modelo centrado na doença e no hospital a um voltado para a pessoa, família e comunidade. Está em jogo uma compreensão ampliada do processo saúde-doença e da necessidade de intervençóes que ultrapassem as práticas curativas. Essa nova forma de cuidado (Ayres, 2004) exige a construção de respostas compartilhadas entre diferentes saberes e setores, atendendo à integralidade desse cuidado. Para tanto, a ideia de Redes de Atenção à Saúde (RAS) se faz imprescindível.

Nesse circuito, a AB não apenas é a porta de entrada prioritária da rede, como também é sua função fazer a coordenação desse cuidado, costurando o percurso desse sujeito em todos os dispositivos que a compóem. Essas unidades estão mais próximas da população e têm maiores chances de observar e avaliar os casos de sobrepeso e obesidade presentes no seu território, a partir da Vigilância Alimentar e Nutricional (VAN), que fazem parte da rotina nesses espaços. Isso gera indicadores importantes do estado nutricional dos residentes daquele território, favorecendo a abor- 
dagem das questóes que envolvem a obesidade e o planejamento de açôes em nível individual, familiar e comunitário.

O perfil epidemiológico atual mostra o quão urgente e importante se torna a formulação e implementação de políticas públicas eficazes e adequadas para a prevenção e tratamento do sobrepeso e obesidade, de modo que reduzam a prevalência nas próximas décadas. Nesse cenário, $\mathrm{AB}$ está no "centro da comunicação", ou seja, na linha de frente, identificando e planejando diferentes açóes, e acionando, quando pertinente, os pontos de atenção secundário e terciário, além de outros setores, a fim de contribuir para um cuidado integral e, portanto, eficiente (Mendes, 2012).

\section{Tecendo a Rede...}

Assegurado pela Constituição de 1988, o Sistema Único de Saúde (SUS) é uma política de Estado que institui a saúde como direito de todos e define princípios e diretrizes para assegurar à população o acesso e cuidado nessa área de forma descentralizada, com a participação comunitária e integralidade do cuidado. Isso implica repensar as antigas estruturas e formas de gestão, vislumbrando a construção e consolidação de uma nova organização da saúde no Brasil de forma articulada que demanda açóes intersetoriais e coordenadas num formato de rede.

A reorganização do modelo fragmentado de atenção à saúde que persistiu ao longo dos anos foi formalmente encaminhada apenas em 2010, quando, por meio da portaria n. ${ }^{\circ} 4.279$, o MS propôs as diretrizes para estruturar a Rede de Atenção à Saúde (RAS) e, com ela, aperfeiçoar o funcionamento político-institucional do SUS, a fim de assegurar açôes e serviços eficientes que garantam a oferta de um cuidado integral.

A proposta de RAS se baseia em "arranjos organizativos de açôes e serviços de saúde, de diferentes densidades tecnológicas, 
que, integradas por meio de sistemas de apoio técnico, logístico e de gestão, buscam garantir a integralidade do cuidado" (Mendes, 2012, p. 49). Sendo o seu objetivo principal promover a integralidade do cuidado, as ações devem ser cooperativas e interdependentes. Sua estrutura operacional se baseia em uma série de componentes, como apoio logístico, centros de comunicação e representantes da governança da Rede de Atenção à Saúde. Eles atuam de maneira a garantir o funcionamento da rede, integrando os diferentes atores envolvidos no processo, bem como os instrumentos necessários para apoiá-los (Mendes, 2011; 2012).

Segundo Duncan et al. (2014, p. 62), a resolutividade no nível da $\mathrm{AB}$ pode chegar:

a $90 \%$ se os recursos financeiros, materiais e humanos forem adequados. Quando o número de encaminhamentos é muito pequeno, isso pode significar que o serviço está deixando de reconhecer situações que são atribuições dos especialistas. Ao contrário, quando esse número é alto demais, por alguma razão os serviços de Atenção Básica estão deixando de exercer todas as suas potencialidades.

No contexto das RAS, as LCs são apresentadas como estratégias que buscam estimular a integração entre os diferentes serviços assistenciais da rede (Giovanella, 2004) e tem como princípio básico "a responsabilização do profissional e do sistema pela saúde do usuário" (Malta e Merhy, 2010, p. 595). Nesse sentido, a LC passa a ser desenhada também no campo da gestão, articulando intervenção nos determinantes sociais e dispondo de tecnologias e instrumentos capazes de impactar o processo saúde-doença (Malta e Merhy, 2010), porém partindo do lugar do singular no ato do cuidado, que só o trabalho vivo (Merhy, 2002) pode dar conta.

Construir uma LC significa, na prática, instituir diretrizes que orientem fluxos assistenciais para a atenção à saúde com intervençóes que envolvam a promoção, prevenção, recuperação, reabilitação, 
incluindo as paliativas. Isso deve se dar num processo constante de comunicação entre os diferentes pontos de atenção à saúde e no suporte tanto dos sistemas logísticos (regulação, transporte etc.) quanto dos de apoio (farmacêutico, teleassistência etc). No entanto, as LCs não devem se limitar à construção de um modelo de procedimentos e protocolos, e sim ao reconhecimento de que os gestores dos serviços podem pactuar fluxos e reorganizar o processo de trabalho, facilitando o acesso do usuário às unidades e serviços dos quais necessita e sobretudo garantir a integralidade do cuidado (Brasil, 2014b). A atuação na macro e micropolítica deve ocorrer de forma articulada, orientada pelas necessidades dos usuários.

Apesar da expressão "Linha de Cuidado", suscitar a imagem de uma sequência coordenada de pontos assim como apresenta a geometria, na prática as LCs nem sempre seguirão a ideia de uma linha como um caminho reto e único, composto por etapas. Ela pode ser representada em muitos casos como uma "trama" ou "conjunto de fios entrelaçados", e esse trançado envolve muitas mãos.

A epidemiologia denuncia um problema e indica a necessidade de organização para solucioná-lo. As discussóes e as pesquisas na área acabam por colocar a obesidade no rol das preocupaçóes de países, sensibilizando-os na construção de programas e políticas públicas que dão suporte legal para as ações. Porém, estas só podem ser implementadas se os profissionais também estiverem sensíveis e puderem usar no cotidiano do seu trabalho ferramentas e tecnologias adequadas, dispondo de uma rede de serviços eficiente e interligada.

Dessa maneira, fazer uma LC funcionar depende da corresponsabilização nos diferentes pontos de atenção à saúde, da existência de sistemas de apoio e logístico e especialmente de suporte legal para as açóes de saúde. Para isso, é importante conhecer os avanços das discussões sobre a obesidade no Brasil e as bases que subsidiaram as atuais diretrizes para a construção da LC, e, com isso, apoiar profissionais e gestores na implementação da LC da obesidade. 


\section{A obesidade e seus nós}

Até a década de 1980, as preocupaçóes do governo federal ainda se dirigiam aos problemas de desnutrição com diversos programas voltados para assistência alimentar e nutricional ao grupo materno infantil e aos escolares. Apenas em 1989, foram divulgados os resultados da PNSN, que, juntamente a outras publicaçóes do período, começou a alertar para a expressiva redução da prevalência da desnutriçáo, ao mesmo tempo em que aumentava a incidência da obesidade. Uma década depois, no ano de 1999, foi aprovada a Política Nacional de Alimentação e Nutrição (PNAN), mostrando a preocupação com a segurança alimentar e nutricional da população brasileira adulta e a prevalência de problemas de saúde relacionados ao sobrepeso e obesidade.

No ano de 2011, a atualização da PNAN inclui a discussão das diretrizes para a melhoria das condiçôes de alimentação, nutrição e saúde da população brasileira com base na articulação com as demais políticas, programas e açôes do SUS (Brasil, 2012a). Decorridos aproximadamente vinte anos desde a sua primeira publicação, muitos desafios para a implementação da política ainda persistem. Destacam-se, dentre eles, as medidas para controle e regulaçáo dos alimentos e o fortalecimento do Sistema Nacional de Segurança Alimentar e Nutricional (SISAN), que ao atuar em cooperação com o SUS contribui para o apoio das açôes de alimentação e nutrição na Rede de Atenção à Saúde. Entre os avanços da PNAN, estão a organização do processo de trabalho, financiamento das açôes e maior controle social. Além disso, os avanços também incluíram o desenvolvimento das açóes de VAN, a construção da agenda de promoção da alimentação saudável, a capacitação de recursos humanos e a produção regular de informaçóes sobre o estado nutricional a partir de pesquisas de base populacional, embora ainda exista o desafio da identificação das iniquidades geográficas e em diferentes grupos populacionais (Recine e Vasconcellos, 2011). 
No mesmo ano da publicação da 2. a edição da PNAN, foi publicado o Plano de açóes estratégicas para o enfrentamento das DCNT no Brasil 2011-22, com o objetivo de preparar o sistema de saúde e incentivar o planejamento de açóes para a redução da prevalência das DCNT até 2022. Esse plano apresenta doze metas, entre elas a redução da prevalência de obesidade em crianças e adolescentes e a estagnação do crescimento da obesidade em adultos, além de outras metas relacionadas à prática de atividade física e alimentação saudável (Brasil, 2011).

Em 2013, foi publicada a Portaria n. ${ }^{\circ}$ 252/GM/MS que instituiu a Rede de Atenção à Saúde das Pessoas com Doenças Crônicas no âmbito do SUS (Brasil, 2013a), atualizada pela Portaria n. ${ }^{\circ}$ 483/GM/MS, em 2014 (Brasil, 2014a). Esses documentos apresentam os princípios e objetivos da RAS, assim como os componentes e competências de cada esfera de gestão, e, portanto, embasaram a publicação e discussão das diretrizes da Portaria n. ${ }^{\circ}$ 424/GM/MS, de 19 de março de 2013, que redefine as diretrizes da organização da prevenção e do tratamento do sobrepeso e da obesidade como linha de cuidado prioritária (Brasil, 2013b), e da Portaria n.o 425/GM/MS, de 19 de março de 2013, que estabelece regulamento técnico, normas e critérios para a assistência de alta complexidade ao indivíduo com obesidade (Brasil, 2013c).

Em 2015, foi instituído o Pacto Nacional pela Alimentação Saudável com o objetivo de ampliar as condiçóes de oferta, disponibilidade e consumo de alimentos saudáveis e prevenir o sobrepeso, a obesidade e as doenças relacionadas à má alimentação da população brasileira. O Pacto prevê a elaboração de um plano de trabalho detalhado com compromissos descritos para execução nas diferentes esferas de governo, com diretrizes e eixos que envolvem ações desde o fortalecimento das políticas de comercialização e de abastecimento da agricultura familiar à defesa do direito à alimentação saudável e adequada, e articulação de açóes para o enfrentamento do sobrepeso, da obesidade e das doenças decorrentes da má alimentação (Brasil, 2015). 
Toda esta base legal expóe o esforço do MS e articulação com demais setores para conter o avanço das DCNT no país. Atualmente há açóes propostas no campo da promoção da saúde, alimentação saudável e prática de atividades físicas, como a Academia da Saúde, o Amamenta e Alimenta Brasil, o Programa Saúde na Escola, e a própria VAN. O desafio que se coloca é implementar de forma eficiente as estratégias sinalizadas nos documentos.

Nesse sentido, o Caderno de Atenção Básica, revisado e publicado em 2014, visa subsidiar os profissionais da $A B$ no atendimento ao sujeito com obesidade e sobre as estratégias de abordagem e o tratamento da obesidade em todos os ciclos de vida. Em 2014, também foi publicado um Manual Instrutivo para a Organização Regional da Linha de Cuidado do Sobrepeso e da Obesidade na Rede de Atenção à Saúde das Pessoas com Doenças Crônicas (Brasil, 2014b).

Essas publicaçôes, em conjunto com as diretrizes estabelecidas nas Portarias n. ${ }^{\circ} 424$ (Brasil, 2013b) e n. ${ }^{\circ} 425$ (Brasil, 2013c), constituem importantes referenciais teóricos e operacionais para gestores e profissionais organizarem a LC do sobrepeso e da obesidade nas secretarias estaduais e municipais de saúde. Elas sinalizam as competências e legitimam o papel de cada esfera na LC, esclarecendo que a Atenção Básica e a Atenção Especializada possuem açóes complementares, e não substitutivas, reforçando a importância das açôes intersetoriais e de articulação nos diferentes pontos de atenção.

\section{A linha de cuidado da obesidade e o papel da Atençáo Básica}

A construção e implementação da LC em obesidade preconiza o planejamento e a organizaçáo das açóes de forma integrada, superando a oferta do cuidado dentro de programas isolados. Para isso, há uma previsão de açôes para as secretarias estaduais e municipais de saúde de modo que sejam complementares e favo- 
reçam a oferta de um cuidado integral ao usuário com obesidade, observando-se a gravidade de cada caso e em nível populacional.

O sujeito que circula nessa rede apresenta especificidades quanto ao desenvolvimento do quadro da obesidade, fatores contribuintes para o ganho de peso que precisam ser escutados e devidamente acolhidos. Esse atendimento atento permitirá a construção de um Projeto Terapêutico Singular (PTS), resultado do planejamento e organização do cuidado em saúde construído entre a equipe e o usuário, considerando as singularidades do sujeito e a complexidade de cada caso (Brasil, 2008; 2009). Tal conjunto ajudará cada sujeito a caminhar nessa LC (Brasil, 2008).

A gravidade da obesidade e das comorbidades associadas podem indicar qual ponto da RAS e conjunto de tecnologias é mais indicado. A avaliação dos riscos e consequentemente o tempo-resposta necessários ao "caso a caso" fazem com que ora o indivíduo receba cuidado em um ponto de atenção, ora em outro, ora em vários ao mesmo tempo. $\mathrm{O}$ imprescindível nesse contexto é que a $\mathrm{AB}$, como porta de entrada prioritária nessa rede e centro de comunicação da RAS, assuma seu papel de ordenadora do cuidado (Mendes, 2012). Com isso, exerce uma função que vai além de "ser parte" da rede, mas é principalmente a agulha que a costura, que articula e coordena o percurso desses sujeitos nessa LC.

No entanto, alguns pressupostos precisam estar bem definidos nesse cenário. Para a $\mathrm{AB}$ atuar como coordenadora do cuidado, a rede precisa estar organizada para receber os sujeitos. A definição do papel de cada ponto de atenção à saúde na LC deve estar bem clara e pactuada, bem como a comunicação entre eles ocorrer de forma constante. É importante ter um fluxo de referência e contrarreferência e é necessário contar com ferramentas de apoio de como e quais critérios serão utilizados para avaliar esses sujeitos e suas necessidades, e, com isso, definir açóes.

Aos municípios que são o lócus de organização, execução e gerência dos serviços e açóes de $A B$, e por consequência das açóes 
aqui discutidas, compete o planejamento e execução das ações (individuais e coletivas) e a oferta dos serviços necessários para o cuidado ambulatorial dos usuários com obesidade, considerando o perfil epidemiológico, os serviços disponíveis, a base territorial, as necessidades de saúde locais e o acesso aos exames, insumos e medicamentos necessários para o tratamento da obesidade no SUS. Os municípios também podem pactuar a LC regional em articulação com outros municípios, estabelecendo o fluxo e a regulação intra e intermunicipal das açóes e dos serviços da RAS (Brasil, 2014c).

De forma geral, as ações da $\mathrm{AB}$, realizadas principalmente por meio da ESF, deveriam incluir, no âmbito individual e coletivo, o acolhimento adequado, ações de VAN, ações de promoção da saúde nos territórios, apoio ao autocuidado, assistência terapêutica multiprofissional com o suporte dos Núcleos Ampliados de Saúde da Família (NASF), inclusive no acompanhamento pós-operatório, e o encaminhamento a outros pontos de atenção à saúde quando esgotadas as possibilidades na $\mathrm{AB}$. As açóes de VAN permitem a identificação da situação alimentar e nutricional, a classificação de risco e gravidade para organização da atenção à obesidade, além de contribuir na avaliação do impacto das açôes desenvolvidas e acompanhar a evolução do estado nutricional do sujeito, sua família e comunidade (Brasil, 2014c).

A Atenção Especializada se divide em três componentes: o ambulatorial especializado, o hospitalar e de urgência/emergência. Ao primeiro, cabe prestar apoio matricial às equipes de $A B$, oferecer assistência aos sujeitos com obesidade quando houver demanda da equipe de referência, avaliar e realizar a indicação para procedimento cirúrgico quando necessário, prestar assistência pré e pós-operatória, organizar o retorno dos usuários à assistência na $\mathrm{AB}$ e realizar contrarreferência em casos de alta, bem como comunicar periodicamente os municípios e as equipes de saúde acerca dos usuários que estão em acompanhamento. O componente hospitalar deve: avaliar os encaminhamentos recebidos com indicação para 
cirurgia bariátrica e realizá-la naqueles que apresentem as condições previstas nos atos normativos; oferecer acesso à cirurgia reparadora e acompanhamento multiprofissional pós-operatório; e organizar o retorno para os serviços de $\mathrm{AB}$. O terceiro corresponde aos serviços de urgência e emergência que podem acometer todo e qualquer sujeito, esteja ele com obesidade ou náo.

É possível perceber que um aspecto que atravessa todos os pontos de atenção à saúde é a importância da comunicação. Os serviços e profissionais envolvidos na LC deveriam se ocupar com o monitoramento das pessoas em acompanhamento, aquelas que se afastaram, bem como os resultados obtidos, estejam eles dentro do esperado ou não. Isso gera um conjunto de dados que são avaliativos não apenas dos serviços em si, mas também permitem que tanto os encaminhamentos quanto as contrarreferências sejam mais bem construídas e eficientes no seu endereçamento. Muito se fala que a $A B$ deve assumir seu papel de ordenadora da rede, mas para que isso aconteça os outros dispositivos devem entendê-la da mesma forma e retornar a ela informaçóes desses usuários, num processo de corresponsabilidade do cuidado, garantindo um efetivo trabalho em rede. Para assegurar um melhor funcionamento, os pontos de atenção à saúde e a descrição da carteira de serviços ofertadas por cada um na LC devem ser definidos e discutidos com os profissionais de modo que cada um tenha consciência da importância do seu papel.

Nesse processo, é importante a criação de sistemas de avaliação e de compartilhamento de informaçóes que facilite a comunicação. Sem ela, a rede não consegue se estruturar, e, provavelmente, cada serviço funcionará à sua maneira e deixará que o percurso seja de responsabilidade de cada sujeito, como se tudo dependesse do seu desejo pessoal. Esse ainda é um nó, pois, na ausência dessa responsabilização e de instrumentos eficientes para agilizar o contato entre os serviços, o usuário, que deveria ter clareza do caminho a 
percorrer nessa LC, acaba por se perder e ter o seu cuidado fragilizado, ferindo seu direito à saúde integral.

Outro ponto transversal a todos os serviços é a questão do acesso aos equipamentos de saúde, que muitas vezes contribuem para a exclusão e estigmatização dos usuários com obesidade, em especial, os mais graves. Esses últimos vivenciam grandes constrangimentos quando o mobiliário e instrumentos não atendem às suas necessidades por não sustentarem seu peso. Quando não há uma estrutura que ofereça inclusão deles, todo o cuidado fica prejudicado. É fundamental, portanto, que a estrutura física, de mobiliário e instrumentos possam favorecer a aproximação desses sujeitos aos serviços (Brasil, 2014a). Isso faz parte de um acolhimento adequado e contribui para a formação de vínculo entre usuário e profissionais, independentemente do ponto de atenção onde esteja. Vale lembrar que as açóes não se resumem à oferta de consultas, mas engloba o acesso regular a exames e medicamentos, bem como às práticas integrativas e complementares, apoio matricial, e acesso imediato aos demais serviços e pontos da RAS, quando necessário (Brasil, 2014b).

Apesar de não ser exclusivo da $\mathrm{AB}$, o vínculo é uma tecnologia leve bastante preconizada, que, somada à escuta, permite pensar o cuidado na longitudinalidade do tempo. Mesmo em casos de encaminhamentos a especialistas, esse usuário ainda mantém vínculo com a sua unidade básica de saúde, sob a responsabilidade da sua equipe de referência, inclusive porque a obesidade é apenas um aspecto da vida desse sujeito e nem sempre aparece como prioridade para ele. É comum que as queixas girem ao redor das comorbidades, principalmente pelas limitaçóes físicas que o excesso de peso lhes impóe, e não na questão do peso e da obesidade em si.

A problemática da obesidade pode aparecer como uma queixa pessoal ou emergir como questão do profissional de saúde que o está atendendo, seja ele profissional da $\mathrm{AB}$ ou especializada, visto a variedade de complicaçóes que esses sujeitos costumam apresen- 
tar. Mas, de forma geral, esses sujeitos iniciam o cuidado na $A B$, pela facilidade de acesso, pelo maior contato com os usuários do território. A atuação das equipes do NASF, por meio do apoio matricial e interconsultas, potencializa as açóes realizadas pela ESF e contribui para o aumento da resolutividade, além de reduzir substancialmente os encaminhamentos equivocados aos demais pontos da rede, impedindo peregrinaçóes desnecessárias pelos serviços e otimizando os recursos em saúde. Franco e Junior (2003) destacam que parte dos encaminhamentos feitos por profissionais da $\mathrm{AB}$ a especialistas não esgota todos os recursos assistenciais disponíveis. Isso é frequente quando a abordagem insiste em focar nos atos prescritivos e na produção de procedimentos, como se estes fossem sinônimos de qualidade de assistência, em vez de estar centrada no sujeito e nas suas necessidades.

A partir dessas consideraçóes, propóe-se o fluxo para a LC em obesidade, com destaque para a $\mathrm{AB}$, exercendo seu papel de porta de entrada e ordenação da rede. 


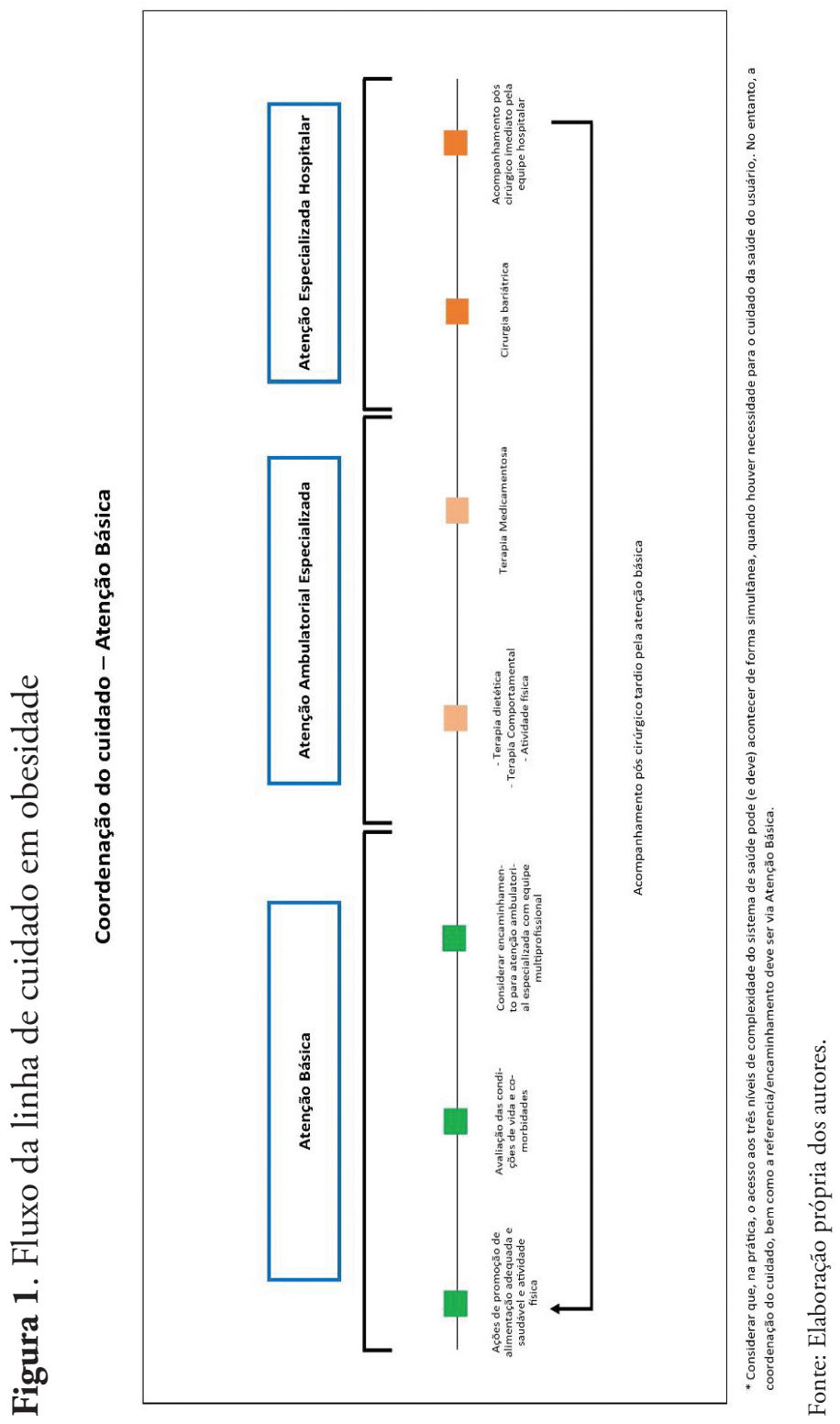




\section{Atribuiçóes dos profissionais da $\mathrm{AB}$}

Embora a VAN não seja competência exclusiva da $\mathrm{AB}$ e possa ser realizada em todos os pontos de atenção da rede, a estratificação das açóes de cuidado em saúde de acordo com o nível de gravidade do IMC é proposta pelo MS, a fim de definir as açôes mais propícias em cada ponto, seguindo o quadro clínico e comorbidades apresentadas.

Os dados obtidos, se sistematizados, permitem o diagnóstico situacional local e planejamento de açôes específicas no território em termos de promoção de alimentação saudável, prática de atividade física e mudança de estilo de vida (MEV). Essas ações tendem a impactar positivamente aqueles indivíduos que, apesar de apresentarem menor risco, como os com sobrepeso ou obesidade grau I, são também mais numerosos. Mas não se pode perder de vista que açóes mais específicas precisam ser implementadas de forma a reduzir a morbimortalidade e melhorar a qualidade de vida por meio de intervençôes clínicas específicas naqueles sujeitos com níveis de obesidade mais graves.

A figura 2 apresenta a proposta do MS para a oferta de cuidado na $\mathrm{AB}$ para indivíduos adultos, conforme classificação do IMC. 


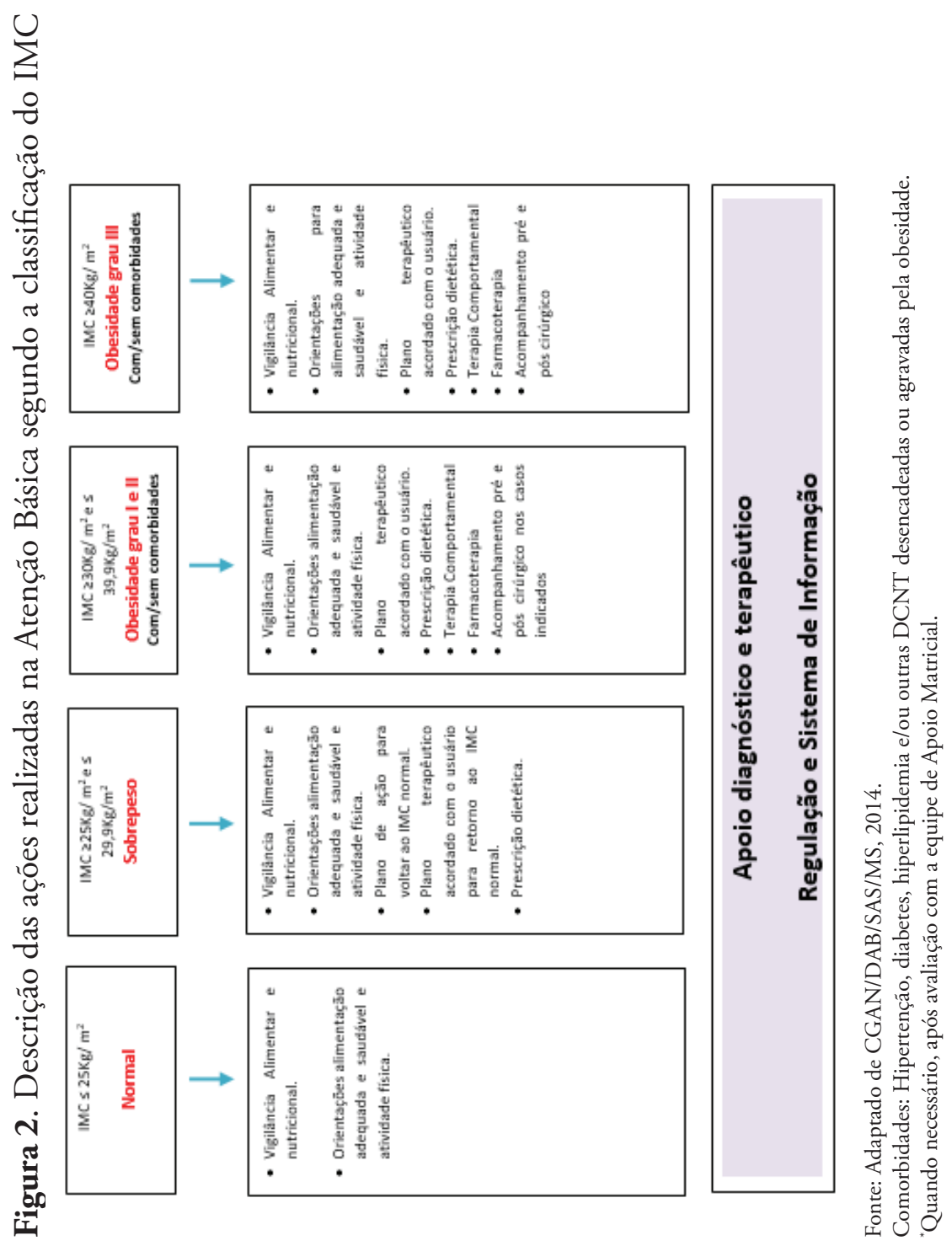


Identificados os casos de obesidade, o profissional de saúde deve, além da avaliação antropométrica, investigar a presença de doenças ou uso de medicamentos que provoquem ganho de peso e avaliar a gravidade, as comorbidades e os fatores de risco de forma a definir o manejo terapêutico de cada caso. A consulta deve ser acompanhada de uma anamnese que inclua não apenas a queixa principal, mas também a motivação para a perda de peso, assim como os hábitos alimentares e de atividade física. Além disso, deve-se estar atento aos aspectos psicológicos associados à obesidade, bem como à presença de transtornos psiquiátricos associados, sejam eles os alimentares, de humor ou outros.

Nos sujeitos com IMC $\leq 30 \mathrm{~kg} / \mathrm{m}^{2}$ sem comorbidades, as açôes devem ter como foco a redução do peso por meio de MEV. Aos sujeitos com IMC entre 30 e $40 \mathrm{~kg} / \mathrm{m}^{2}$, é colocada a prioridade de perda de peso de 5 a $10 \%$, visto que a reduçáo dos riscos associados pode ser observada já nessa faixa. Àqueles com IMC $\geq$ $40 \mathrm{~kg} / \mathrm{m}^{2}$, é necessária a perda acima dos $10 \%$ do peso (Duncan et al., 2014). Em todos os casos, o seguimento deve ser feito de forma a monitorar a situação, as limitaçóes e os avanços. Para isso, é preciso reavaliá-los dentro de um determinado espaço de tempo a ser estipulado frente às especificidades de cada caso.

É nesse processo de avaliação, monitoramento e reavaliação que o profissional da $\mathrm{AB}$ pondera a necessidade de acionar outros profissionais e dispositivos da rede, fornecendo um leque de opçôes não apenas viáveis, mas também sustentáveis pelo sujeito. As equipes NASF são as mais próximas e realizam atividades de promoção de saúde no território, funcionam como articuladores no processo de cuidado e oferecem apoio técnico às equipes da ESF no cuidado desses usuários. De forma geral, as equipes NASF são definidas pelos gestores municipais, considerando o diagnóstico epidemiológico local, os critérios de prioridades definidos e a rede de serviços disponíveis (Brasil, 2012b; 2014b). Entretanto, é preciso refletir quais seriam as categorias profissionais que mais ofertariam 
suporte ao manejo da obesidade na $\mathrm{AB}$ e na Atenção Especializada ambulatorial.

As categorias profissionais mais encontradas são: médico endocrinologista, nutricionista, enfermeiro, educador físico, psicólogo, assistente social e fisioterapeuta. As açóes realizadas por cada profissional são baseadas em disposiçóes legais que regulamentam o exercício de cada uma das profissóes, porém, destaca-se a importância do olhar ampliado à multifatorialidade e complexidade da obesidade e o trabalho em equipe. É preciso que o profissional compreenda o contexto de vida do sujeito, partindo de uma lógica náo culpabilizante, compreendendo os agravos em saúde como consequências de condiçóes sociais adversas que contribuem para o aumento do peso e das possíveis dificuldades para a perda.

Contudo, embora o profissional tenha tal compreensão, ele não deve tirar de si a responsabilidade de um cuidado compartilhado com o sujeito. Este traz a necessidade da negociação, na qual o cuidado não é imposto, mas oferecido e pactuado, considerando os desejos e escolhas do indivíduo, assim como os seus modos de vida, o já referido PTS (Brasil, 2008; 2009).

\section{Consideraçóes finais}

A obesidade tem se apresentado como um crescente problema de saúde nos últimos anos e mobilizado países a construírem diretrizes para o cuidado do sujeito com obesidade, principalmente pelos custos e sobrecarga do sistema de saúde. Por isso, é imprescindível a construção de linhas de cuidado que definam os fluxos assistenciais nos estados e municípios, preconizando açóes complementares e interligadas e que contribuam para oferta de cuidado integral ao usuário com obesidade.

O MS define as atribuiçóes referentes a cada ponto de atenção à saúde e parte do pressuposto que eles devam integrar as RAS. Para isso, gestores e profissionais devem estar sensíveis a essa questão 
e compreender suas responsabilidades em todo o processo. Isso inclui a coleta de dados, diagnósticos situacionais, planejamento de políticas públicas, publicações específicas para profissionais, formação e capacitação de especialistas na abordagem do sujeito com obesidade, bem como as açóes voltadas para seu tratamento.

Neste capítulo, coube o recorte da $\mathrm{AB}$, entendendo que ela é a organizadora da rede e que, por isso, ela seria a agulha que a costura. Além disso, ela valoriza açóes que se apoiam em tecnologias de cuidado leves, baseadas no vínculo, acompanhamento longitudinal e abordagem centrada na pessoa. Sendo assim, ela se esforça para superar a produção de procedimentos como forma única de prática profissional e se propóe a trabalhar em corresponsabilidade com o sujeito e outros atores que se façam necessários na construção de um PTS.

No plano ideal, essa é uma configuração extremamente rica e eficiente, porém, a prática esbarra em muitos problemas. A primeira delas talvez seja a própria formação de profissionais no modelo tradicional, que atribui maior importância às tecnologias duras e dialogam menos com os sujeitos que, de fato, vivenciam não apenas o quadro das doenças, mas também as dificuldades e complicaçóes dela decorrentes.

Parece ser a escuta atenta e qualificada que permite entender os diferentes e complexos processos que envolvem o ganho de peso e acolhe as queixas relacionadas às dores físicas e emocionais a ela associadas. Cada sujeito apresenta uma história que, obviamente, é singular, vinculada a uma história familiar e uma história social, e essa complexidade deve compor os planos de intervenção.

No entanto, apesar de muito prevalente, a obesidade ainda carrega uma representação social negativa, vinculada a um julgamento moral que impacta esse processo de escuta. Os sujeitos com obesidade moderados a graves sofrem com estigmas, como se sua condição fosse decorrente de falta de vontade, falta de controle, falta de confiança. E é bastante possível que esse seja um dos motivos que 
faça o transtorno ainda não compor os grupos prioritários da $\mathrm{AB}$. Ele está muito presente no cotidiano das unidades, porém, arrisca-se dizer que, na maior parte das vezes, aparece como coadjuvante na abordagem de comorbidades, como hipertensão e diabetes.

Apesar dos grandes desafios que se colocam na $A B$, na qual, muitas vezes, os profissionais estão consumidos pelo excesso de demanda e sobrecarga de trabalho, reconhece-se o papel primordial que ela ocupa na rede e o quanto é importante reunir esforços no campo da gestão para seu devido empoderamento.

\section{Referências}

AMERICAN SOCIETY FOR BARIATRIC SURGERY. "Guidelines for reporting results in bariatric surgery. Standards Committee". Obesity surgery, v. 7, n. 6, pp. 521-2, dez. 1997.

AYRES, J. R. de C. M. "O cuidado, os modos de ser (do) humano e as práticas de saúde”. Saúde \& Sociedade, v. 13, n. 3, pp. 16-29, 2004.

BAHIA, L. R. e ARAÚJO, D. V. "Impacto econômico da obesidade no Brasil". Revista HUPE, v. 1, n. 13, pp. 13-7, Rio de Janeiro, 2014.

BRASIL. Ministério da Saúde. Política Nacional de Humanização. Clínica Ampliada, equipe de referência e projeto terapêutico singular. 2 ed. Brasília: Ministério da Saúde, 2008.

. Ministério da Saúde. Política Nacional de Humanização da Atenção e Gestão do SUS: gestão participativa e cogestão. Brasília: Ministério da Saúde, 2009. . Ministério da Saúde. Portaria n. ${ }^{\circ}$ 4.279, de 30 de dezembro de 2010. Estabelece diretrizes para a organização da Rede de Atenção à Saúde no âmbito do Sistema Único de Saúde (SUS). Brasília: Ministério da Saúde, 2010.

. Ministério da Saúde. Plano de açôes estratégicas para o enfrentamento das doenças crônicas não transmissiveis (DCNT) no Brasil 2011-2022. Brasília: Ministério da Saúde, 2011.

. Ministério da Saúde. Política Nacional de Alimentação e Nutrição. Brasília: Ministério da Saúde, 2012a. (Série B. Textos Básicos de Saúde).

- Ministério da Saúde. Política Nacional de Atenção Básica. Brasília: Ministério da Saúde, 2012b.

. Ministério da Saúde. Portaria n. ${ }^{\circ} 252$, de 19 de fevereiro de 2013. Institui a Rede de Atenção à Saúde das Pessoas com Doenças Crônicas no âmbito do Sistema Único de Saúde (SUS). Brasília: Ministério da Saúde, 2013a. 
. Ministério da Saúde. Portaria n. ${ }^{\circ} 424$, de 19 de março de 2013. Redefine as diretrizes para a organização da prevenção e do tratamento do sobrepeso e obesidade como linha de cuidado prioritária da Rede de Atenção à Saúde das Pessoas com Doenças Crônicas. Brasília: Ministério da Saúde, 2013b.

. Ministério da Saúde. Portaria n. ${ }^{\circ} 425$, de 19 de março de 2013. Estabelece regulamento técnico, normas e critérios para a Assistência de Alta Complexidade ao Indivíduo com Obesidade. Brasília: Ministério da Saúde, 2013c.

. Ministério da Saúde. Portaria n. ${ }^{\circ} 483$, de $1 .^{\circ}$ de abril de 2014. Redefine a Rede de Atenção à Saúde das Pessoas com Doenças Crônicas no âmbito do Sistema Único de Saúde (SUS) e estabelece diretrizes para a organização das suas linhas de cuidado. Brasília: Ministério da Saúde, 2014a.

. Ministério da Saúde. Secretaria de Atenção à Saúde. Departamento de Atenção Básica. Cadernos de Atenção Básica: Estratégias para o cuidado da pessoa com doença crônica: obesidade, n 38. Brasília: Ministério da Saúde, $2014 \mathrm{~b}$.

- Ministério da Saúde. Organização Regional da Linha de Cuidado do Sobrepeso e da Obesidade na Rede de Atenção à Saúde das Pessoas com Doenças Crônicas - Manual Instrutivo. Brasília: Ministério da Saúde, $2014 \mathrm{c}$.

. Presidência da República. Decreto n. ${ }^{\circ}$ 8.553, de 3 de novembro de 2015. Institui o Pacto Nacional para Alimentação Saudável. Brasília: Casa Civil, 2015. . Ministério da Saúde. Portaria n. ${ }^{\circ} .436$, de 21 de setembro de 2017. Aprova a Politica Nacional de Atenção Básica, estabelecendo a revisão de diretrizes para a organização da Atenção Básica, no âmbito do Sistema Único de Saúde (SUS). Brasília, DF: Ministério da Saúde, 2017.

. Ministério da Saúde. Secretaria de Vigilância em Saúde, Departamento de Análise em Saúde e Vigilância de Doenças não Transmissíveis. Vigitel Brasil 2018: Vigilância de fatores de risco e proteção para doenças crônicas por inquérito telefônico: estimativas sobre frequência e distribuição sociodemográfica de fatores de risco e proteção para doenças crônicas nas capitais dos 26 estados brasileiros e no Distrito Federal em 2018. Brasília, 2019. Disponível em: https://portalarquivos2.saude.gov.br/images/pdf/2019/julho/25/vigitel-brasil-2018.pdf. Acesso em: 12 set. 2019.

CALLE, E. E. et al. "Body-mass index and mortality in a prospective cohort of U.S. adults”. New England Journal of Medicine, v. 341, n. 15, pp. 1097-105, 07 oct. 1999.

DUNCAN, B. B. et al. Medicina ambulatorial: condutas de Atenção Primária baseadas em evidências. 4 ed. Porto Alegre: Artmed, 2014.

FRANCO, T. B. e MAGALHÃES Jr., H. M. "Integralidade na assistência à saúde: a organização das linhas do cuidado". In O trabalho em saúde: olhando e experienciando o SUS no cotidiano, v. 2. 2003, pp. 125-34. 
GIOVANELLA, L. Redes integradas, programas de gestão clínica e clínico geral-coordenador: reformas recentes do setor ambulatorial na Alemanha (relatório de pós-doutorado). Frankfurt am Main: Institut für Medizinische Soziologie, JW Goethe Universität, 2004.

MALTA, D. C. e MERHY, E. E. "O percurso da linha do cuidado sob a perspectiva das doenças crônicas não transmissíveis”. Interface - Comunic., Saúde, Educ., v. 14, n. 34, pp. 593-605, jul./set. 2010.

MERHY, E. E. Saúde: a cartografia do trabalho vivo. São Paulo: Hucitec, 2002. e FEUERWERKER, L. C. M. "Novo olhar sobre as tecnologias de saúde: uma necessidade contemporânea”. In MANDARINO, A. C. S. e GOMBERG, E. (orgs.). Leituras de novas tecnologias e saúde. São Cristóvão: Editora UFS, 2009.

OLIVEIRA, M. L. Estimativa dos custos da obesidade para o Sistema Único de Saúde do Brasil. Brasília: UnB, 2013.

MENDES, E. V. As Redes de Atenção à Saúde, v. 549. Brasília: Organização Pan-Americana da Saúde, 2011.

. O cuidado das condições crônicas na atenção primária à saúde: o imperativo da consolidação da estratégia da saúde da família. Brasília: Organização Pan-Americana da Saúde, 2012.

RECINE, E. e VASCONCELLOS, A. B. "Políticas nacionais e o campo da Alimentação e Nutrição em Saúde Coletiva: cenário atual”. Ciência \& Saúde Coletiva, v. 16, n. 34, pp. 73-9. 2011.

STARFIELD, B. Atenção Primária: equilíbrio entre necessidades de saúde, serviços e tecnologia. Brasília: UNESCO, Ministério da Saúde, 2002.

WORLD HEALTH ORGANIZATION. Physical status: the use and interpretation of anthropometry. Geneva, 1995. (WHO Technical Report Series, n. 854). - Obesity: preventing and managing the global epidemic: report of a WHO consultation, 2000, p. 252.

. World Health Statistics 2012, 2012, p. 176. 